\title{
MRMA: A Multicast Resource Management Architecture for IP Platforms
}

\author{
Ryan $\mathrm{Wu}^{1}$ and Irene Katzela ${ }^{2}$ \\ ${ }^{1}$ ECE Department, University of Toronto, Toronto, Canada \\ ${ }^{2}$ Lucent-Networkcare, Toronto, Canada \\ katzeladlucent.com
}

\begin{abstract}
Multicast with QoS guarantees is a bandwidth-efficient transmission scheme for the delivery of multi-user multimedia applications. Unlike most other related work, which focuses primarily on the algorithmic or protocol aspects of the multicast problem, our work focuses on the architecture and implementation aspects. In this paper, we present MRMA, a multicast resource management architecture designed to provision multicast with QoS guarantees over the IP platform. MRMA offers a flexible framework for integrating the necessary components for implementing the key multicast functions that are needed for creating, maintaining and terminating multicast sessions: QoSsensitive route selection and tree construction, address allocation, and session advertisement. Taking into account network operations that are unique to multicast-with-QoS, MRMA implements a hierarchical architecture that features centralized monitoring of network state and semi-distributed persession multicast management. The architecture also implements the idea of moving the heavy computational load of administering multicast operations outside the network and onto the hosts.
\end{abstract}

\section{Introduction}

The exponential growth of today's Internet is fueled in part by an emerging class of multi-user broadband applications such as video-on-demand, video conferencing, and file distribution. These applications are generally characterized by high transmission bandwidth, high data processing and stringent Quality-of-Service (QoS) requirements. Multicast with QoS guarantees, referred to in this paper simply as multicast-with-QoS or multicast, is an efficient transmission scheme for the delivery of multi-participant multimedia applications with respect to bandwidth resource usage. It emphasizes on minimizing the amount of traffic that is needed for distributing the data to its destinations.

Multicast has sparked deep interest among the research and industry community in the last ten years since Deering first described the standard multicast model for IP networks [1], and there are many research papers written on each of the key multicast topics/functions. We list some of them here. For multicast routing algorithms, there exist many proposed heuristics for the classic Steiner Tree and Constrained Steiner Tree problems [3]. For multicast protocols, there are two categories: QoS-oblivious - 
PIM, CBT, BGMP, DVRMP, MOSPF, and QoS-sensitive - YAM, QoSMIC[6]. For multicast address allocation, there is the suite of IETF protocols that support intradomain and inter-domain address allocation: MASC, AAP, MADCAP, and MAAA. For session announcement, there is the $s d r$ tool and SDP protocol. Lastly, for reliable transport service, there are many proposed multicast transport protocols such as SRM, RMTP-II, PGM, ARM. We would like to note that all the work listed above analyzes the multicast problem from either the algorithmic or protocol aspect, and generally focuses on a one or two topics. In particular, none of them approach the multicast problem as a whole and address multiple multicast topics together.

Our work takes an architectural approach to addressing the multicast problem as a whole. The goal of this paper is to propose an implementable solution that addresses all the key multicast functions. We introduce MRMA, a multicast resource management architecture that is customized for provisioning multicast-with-QoS service over the IP platform. MRMA is not simply a multicast protocol, but rather a management architecture that integrates all of the key multicast components into a single coherent system for managing the setup and configuration of multicast-withQoS connections. The key multicast components are: i) a protocol for conducting QoS-sensitive route selection and tree construction, ii) an addressing scheme for managing the allocation of multicast addresses, iii) a session directory for announcing the presence of active multicast sessions to the network, iv) and a transport protocol for providing reliable delivery of multicast data.

The rest of this paper is organized as follows. Section 2 discusses some related work. Section 3 presents the MRMA architecture and discusses the key design issues. Section 4 suggests some future research directions for on the MRMA.

\section{Related Work}

In this section we present a brief description of MBone, a primitive best-effort multicast architecture. Note however, that it is only somewhat related to MRMA. We are currently not aware of any other proposed architecture that is exactly comparable to the MRMA, that is, a management architecture for provisioning multicast service with QoS guarantees.

Multicast Backbone (MBone). The MBone [2] is an experimental prototype for demonstrating the feasibility and cost-saving advantages (in terms of bandwidth) of the multicast scheme, and is expected to be eventually phased out. It is an over-lay network and utilizes the DVRMP and MOSPF protocols to handle the routing functions; the session directory tool, $s d r$, as the session announcement server; and manual address allocation. This is the only work that we are aware of that, similar to our work, strives to address all the key components necessary for enabling multicast over the Internet. However, unlike MRMA, it can only provision best-effort multicast service.

The fundamental problem is that the MBone model was constrained by the network infrastructure to essentially 'emulate' multicast service over a native unicast-only Internet. It focused on extending and adding onto the existing IP protocol suite, which is specialized for best-effort unicast connections. For instance, the DVRMP 
and MOSPF multicast routing protocols are in fact merely simple extensions of the unicast routing protocols RIP and OSPF respectively. As such, DVRMP and MOSPF do not incorporate any of the QoS-sensitive multicast algorithms described in [3], and consequently the multicast trees they produce can only be expected to satisfy very simple tree constraints or optimizations. Moreover, the MBone is also lacking a connection-admission-control mechanism to regulate access to the network, a resource reservation mechanism that acquires network resources on behalf of the multicast group and reclaims idle resources on behalf of the network, a session manager to track relevant group membership information, and an effective monitoring mechanism that can generate accurate, up-to-date topology information for routing and traffic control purposes - all of which are essential for providing multicast and QoS guarantees. The MRMA architecture, on the other hand, does not suffer the same drawbacks.

\section{MRMA Overview}

In this section we present the design for MRMA, a multicast management architecture customized for provisioning multicast-with-QoS over IP networks. The design takes into consideration the group-centric properties that are unique to multicast in order to maximize the scalability, flexibility and accuracy of the architecture. Unfortunately, due to length restrictions on this paper we can only provide an overview of the architecture here. More detailed specifications are documented in [5]. We begin the discussion by describing the operation context of MRMA: operating platform and provisioned services. Next, we continue with a description of the architecture and discuss the key design decisions. Finally, we show how the MRMA can be applied to provision an example multicast service, a layered video multicast application.

\subsection{Context Model}

As is the case with any network management architecture, a detailed implementation specification of the MRMA is highly dependent on its operational context. In other words, the exact design for the architecture is governed by the context model which specifies the properties of the physical network and the services it supports. As such, in order to facilitate detailed specification of core MRMA features we narrow the scope of the current version of the management system.

We are designing the MRMA to operate over a 'next-generation' IP platform that contains primarily 'intelligent' routers with native support for both unicast and multicast routing, flow-recognition, and local control of CPU and link bandwidth allocation. The routers are also programmable. Such routers eliminate the need to implement IP tunneling, and allows QoS-sensitive packet scheduling and dynamic control of network configuration. We are also assuming each domain is small enough in terms of number of nodes and physical size such that it is feasible to implement some form of centralized monitoring mechanism without the associated congestion or bottleneck problem arising from too much signaling traffic converging at one point. MRMA supports one-to-many intra-domain multicast that adheres to the Express 
Multicast Model [4], which makes fundamental changes to the traditional Deering IP multicast model [1] used in the MBone. Express is a single-source service model that allows only one source to multicast on each group. We would like to stress at this point that although our current MRMA version is customized for intra-domain operation, the general MRMA architecture can be extended to provision inter-domain operation. Suggestions for such extensions are presented in [5]. A detailed description of the MRMA context model is also presented in [5].

\subsection{MRMA Architecture}

From the functional perspective, the architecture can be viewed as comprising of various functional blocks that are 'contained' within a kernel, as illustrated in Fig. 1. We model each of the multicast functions route selection, address allocation, session advertisement and traffic and congestion control as Functional Modules (FMs). The FMs have well-defined input/output specifications and perform very specific functions. This is a feasible approach since each multicast function is unique, and so each FM can be modeled as independent blackboxes whose internal design and implementation do not have a major affect on the design of the rest of the management system as long as it produces the desired output given some input.

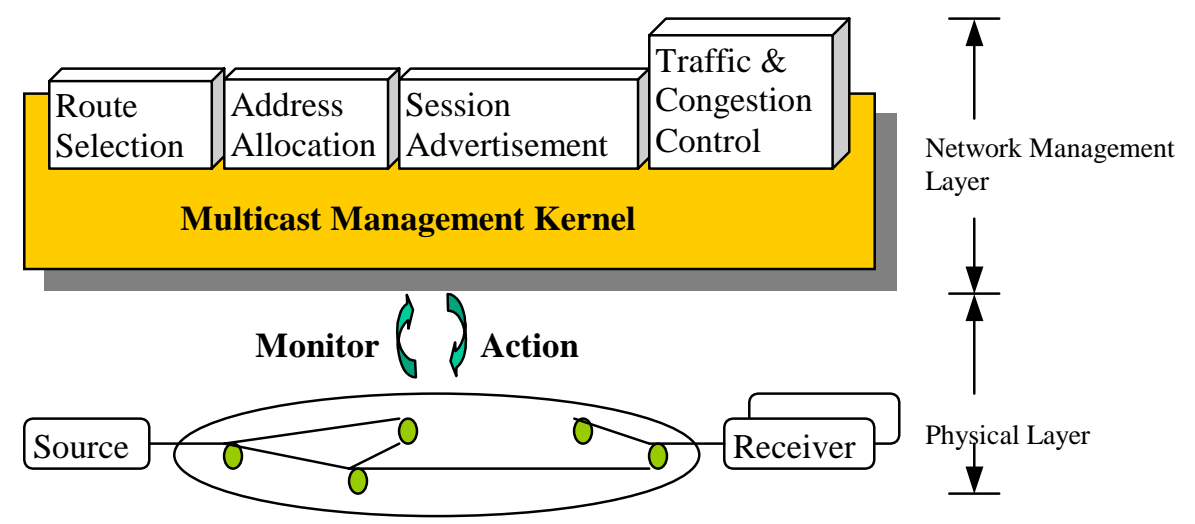

Fig. 1. Functional View of the MRMA Architecture

The Multicast Management Kernel (MMK) is the core component that integrates the various FMs into a coherent system. MMK coordinates the execution of highlevel multicast operations such as creating a new session, adding a new member, etc. by invoking services from the FMs. In return, the kernel supports the FMs by performing housekeeping tasks such as gathering accurate network state information to be used as input for FM decision-making. MMK communicates with the FMs through well-defined interfaces.

The advantage of implementing each of the key multicast functions as modules is that it increases the adaptability of the MRMA architecture. It is designed such that the MMK will be able to swap-in and swap-out, with minimal customization, different solutions for each of the multicast functions. Some of the existing 
implementations and proposals for the key multicast functions listed in Section 1 can be modified to serve as FMs.

From an implementation perspective, the MMK and FM model discussed above is not implemented as a single software entity, but is rather implemented as a set of distributed, hierarchically-interacting management entities: Domain Manger (DM), Session Manager (SM), and Element Agent (EA). The collection of entities control network operation at three levels: there is one DM per domain that manages domain level operations; there is one SM per multicast session that manages session level operations, and there is one EA per network router that manages local node level operations. The entities share a hierarchical manager-agent relationship. One DM manages multiple SMs and EAs, and each SM in turn manages multiple EAs. The EAs manage the local node functions and also serve as agents to the DM and SMs. The function and design of the management entities reflect our desire to develop an architecture that effectively balances two generally conflicting management goals: maximize the distribution of the heavy processing load of managing each multicast session while ensuring the system is still capable of making accurate management decisions. These two goals are conflicting in that, generally, good load distribution is best achieved with a distributed management paradigm while accurate decisionmaking is better with a centralized management paradigm. The architecture for the DM and SM are illustrated in Fig. 2 and Fig. 3., and are described in detail in [5]. The architecture for the EA is not included in this paper, but it is also described in [5].

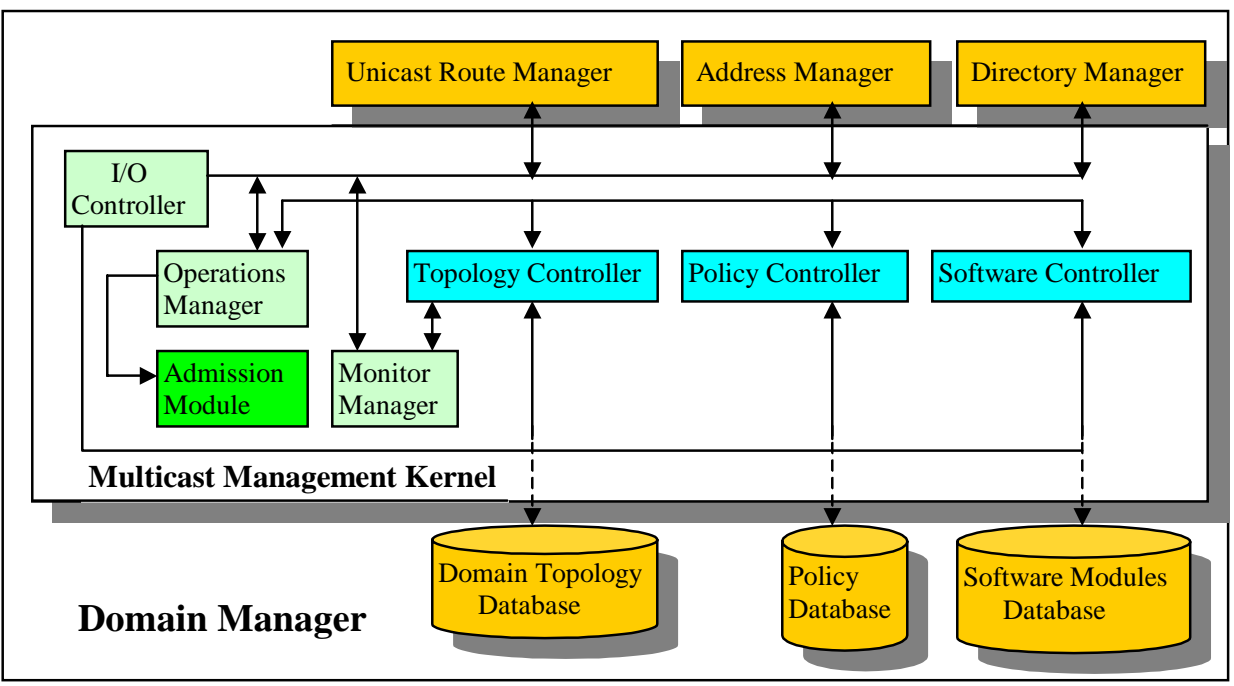

Fig. 2. Domain Manager Architecture

Domain Manager. The Domain Manager implements centralized management of domain-wide operations. The DM architecture is shown in Fig. 2. Some major functions of the DM are: 
- Topology tracking. The primary DM function is to continuously monitor and maintain an accurate, up-to-date image of the domain state. The Monitor Manager uses a combination of explicit polling and 'trap-directed' polling [5] to collect node, link, traffic, and QoS metrics such as bandwidth usage and link congestion from the domain. The status information is then passed to the Topology Controller for storage in the Domain Topology database. The Monitor Manager ensures the collected information is accurate and up-to-date by taking steps to verify the integrity of received status messages. Damaged and out-of-sequence messages are filtered out. Topology image information is used as input for making route selection and admission control decisions.

- Directory service. Multicast session names and the corresponding session addresses, maximum source rate, and session duration are some of the properties tracked by the Directory Manager, a FM which serves a similar functionality as the $s d r$ tool deployed on the MBone.

- Address management. The Address Manager is another FM which controls the allocation of a limited pool of multicast session addresses. It can implement a scheme based on the general Multicast Address Allocation Architecture (MAAA) .

- Administer domain-wide policies. DM acts as a policy server for controlling the distribution of policies and rules that govern domain-wide decisions. For instance, some domain-wide policies may limit the maximum aggregate bandwidth that a session is allowed to consume, or the maximum session duration that a session is allowed to live.

- Inter-domain peer-to-peer communication. The DM is also responsible for exchanging summarized domain topology information with peer DMs in neighboring domains to enable inter-domain multicast operation. Managing multidomain multicast trees that span multiple domains would require sharing of summarized domain information.

We have chosen a centralized management paradigm for handling domain-wide operations because it is generally more accurate in gathering and summarizing topology information, as long as the propagation delay experienced by status reports from the agents to the manager is acceptable, a condition which is assumed to be true in the MRMA context model. By having all the nodes report back to the DM, it will have complete knowledge of all the node and link states. That knowledge can then be used to correlate partial information contained in each report and formulate an accurate picture of the entire domain. The downside is that such a paradigm may suffer from congestion on links near the DM as reports from all the nodes converge. Fortunately, the problem may not be a major since most signaling traffic to the DM will generally be short status report messages. An alternative option is to deploy a semi-distributed paradigm, whereby there are several DMs per domain, and each DM has complete knowledge of a portion of the domain. However, this method suffers from that fact that there is no single source from which other network entities can obtain a full, accurate, up-to-date image of the entire domain.

Since the DM represents a centralized point of convergence for a large volume of signaling traffic, its physical location in the network has an important impact on the performance of both the DM and the nearby network links. Therefore, the DM should reside in the 'center' of the network such that it maximizes the distribution of 
signaling traffic across the network and minimizes the maximum propagation delay experienced by status reports from network nodes [5].

Session Manager. To tailor the MRMA to handle requirements that are unique to multicast, we introduce the notion of a Session Manager. There is one SM dedicated for managing all operations relating to the setup and configuration of each session. SMs are dynamically spawned by the multicast sources to create and manage the multicast sessions. A SM resides on the same host computer as the source and shares the same IP address, but communicates on different ports. The SM architecture is shown in Fig. 3. The two major SM functions are:

- Multicast tree construction. Each SM manages the construction of a multicast tree that spans the source and all the group members. Tree construction involves initializing the session by registering with the DM, grafting new members onto the tree, pruning departing members and terminating the session. We will elaborate on these operations when we introduce our multicast protocol in Section 3.3. Multicast route selection and resource reservation are two important steps in tree construction.

The SM implements a centralized route selection scheme. To add a new member, Rnew, to the group, the SM first invokes the Multicast Route Manager to attempt to compute a feasible route from the existing tree to Rnew. The Route Manager's multicast routing algorithm takes as input the current state of the entire domain, the current configuration of the tree, and the set of application or receiverspecific QoS metrics. The computed route, Path(Src, Rnew) must satisfy the QoS constraints defined by the application or Rnew, where Src is the multicast source. Note that a section of Path(Src, Rnew) coincides with the existing tree (trunk section) while the other section represents the new branch that needs to be grafted (branch section). QoSMIC also introduces the notion of a Manager Router for managing a centralized Multicast Tree Search Procedure [6].

Other than selecting the path, SM is also responsible for reserving resources from all the on-path nodes along the Path(Src, Rnew). Along the trunk section, SM must ensure the on-path nodes reserve additional bandwidth, if required, to accommodate the QoS requirements of the new member. Similarly along the branch section, SM must ensure the nodes reserve sufficient bandwidth and add an entry for Rnew in their multicast routing tables. Once a feasible route is found and resource reservation negotiations are successfully completed, Rnew can begin receiving multicast traffic.

- Multicast tree performance maintenance. Similar to the DM, the SM also has a Monitor Manager that continuously monitors the state of the tree and collect statistics that are needed for performance and connection admission control. For instance, if congestion is building up at certain points on the tree, the SM may reconfigure the entire tree, deny access to new members, or lower the source transmission rate to alleviate the problem.

The SM maintains two topology databases: Tree Topology which tracks group membership and tree topology information, and Domain Topology which tracks node and link state information for the entire domain. Each SM maintains 
its own Tree Topology image since they each manages its own multicast session. On the other hand, changes in the Domain Topology image are periodically downloaded from the DM. The update frequency is chosen such that it ensures that the local copy closely reflects the true network state while avoiding the need for excessive updates from the DM.

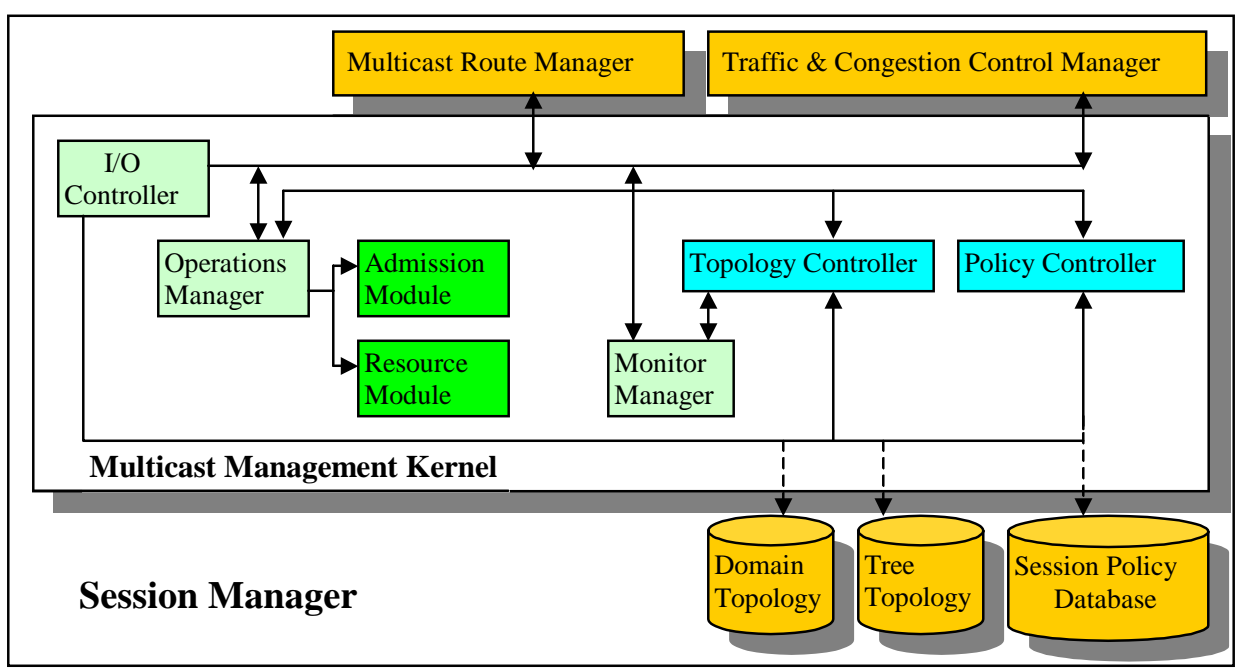

Fig. 3. Session Manager Architecture

Dedicating one SM per session naturally distributes the total computation load among many management entities, while still allowing multicast routing and other session-related decisions to be decided centrally which results in creation of more optimal multicast trees. Multicast connections are more complex and computationally intensive to manage than unicast connections. Other than node and link state information, multicast routing and resource control also require tree structure and group membership information. Thus, to maximize the scalability of MRMA, it is important for the management architecture to maximize the distribution of the aggregate computational load across the network and to minimize the signaling overhead (i.e., eliminate the need for flooding). On the other hand, accuracy of the routing decisions is also important since it determines the efficiency in which network resources are utilized. A connection that is granted more bandwidth than its statistical mean requirement implies there will be less resources available for others, resulting in a non-optimum call-blocking rate. The accuracy of management decisions is dependent on the accuracy and completeness of the knowledge that the manager has of the true state of the network.

A fully distributed architecture may be effective in spreading the computational load across the network, but it is difficult for any one entity to obtain accurate and timely knowledge of the entire network, and so it suffers from the inability to make accurate management decisions. A central architecture, on the other hand, generally has complete knowledge of the entire topology and is therefore able to make more 
optimum routing and management decisions, but it does have the disadvantage of being a single point of congestion. The adverse effect is congestion on links located near the central manager. Moreover, as all network functions are executed at the manager, response times will experience long latencies as requests face long queuing times. MRMA's hierarchical arrangement of having global performance statistics monitored centrally by the DM, while session-specific statistics, which are of no use to other multicast groups, are handled by the corresponding SMs is a good comprise between the above two options. It offers a good balance between accuracy and scalability.

The physical location of each SM also greatly affects the load distribution. Placing all the SMs at a centralized location is definitely ineffective since it simply behaves like a centralized system. For source-based multicast trees, two possible options are: SMs reside on the access routers situated around the network perimeter, or SMs reside outside the network on the actual host/source computer. The first option achieves relatively good network load distribution, but it may still suffer from congestion for the case where an access router is serving many host computers that themselves are roots for different multicast trees. As such, the access router may be managing too many multicast sessions simultaneously and become a potential congestion point. MRMA implements the second option. Deploying the SM at the host computer maximizes the load distribution. In fact, it eliminates the task of setting-up and configuring the multicast session from the network altogether.

Element Agent. There is one Element Agent deployed on each network node. One of EA's primary functions is to manage the allocation of local node and link resources such as bandwidth, including handling resource reservation requests from the SMs. To guarantee Quality-of-Service, each EA tracks all the multicast trees that it is a part of and the amount of node and link resources that are reserved for each multicast flow. With that information, it can then apply the appropriate scheduling service for each flow. The EA's other major role is to monitor local node and link state information and periodically forward the summarized data to the DM and SMs.

Implementing resource management at the node-level evenly distributes the load of managing the network's resources. Moreover, by locating the resource manager close to the actual resource, the EA has all the necessary information needed to make timely and accurate resource allocation, routing and scheduling decisions locally.

\subsection{MRMA Multicast Protocol}

We also developed a QoS-sensitive multicast protocol to accompany the MRMA architecture. The Native MRMA Management Protocol (NMMP) specifies the execution procedures for all the MRMA operations. Note that although the current MRMA version supports NMMP, the general MRMA architecture can be readily customized to support other similar protocols such as QoSMIC [6].

[5] provides a detailed description of the protocol, but we shall introduce it here through an example application (see Fig. 4). We will describe how to multicast layered video over a MRMA-managed network. Layered video multicast protocols such as Receiver-Driven Layered Multicast (RLM) and Layered Video Multicast with Retransmissions (LVMR) generally have a source that transmits video as multiple 
multicast layers/streams. Each receiver can than decide on the number of layers they would like the network to deliver to them, depending on its processing capacity. The more layers a receiver receives, the better the picture quality. For our example, the Src is the multicast source and $\mathrm{R} 1 \ldots \mathrm{Rx}$ are the receivers. The video is encoded into four layers L1...L4. Depending on the QoS desired, the receivers request the appropriate number of layers to receive.

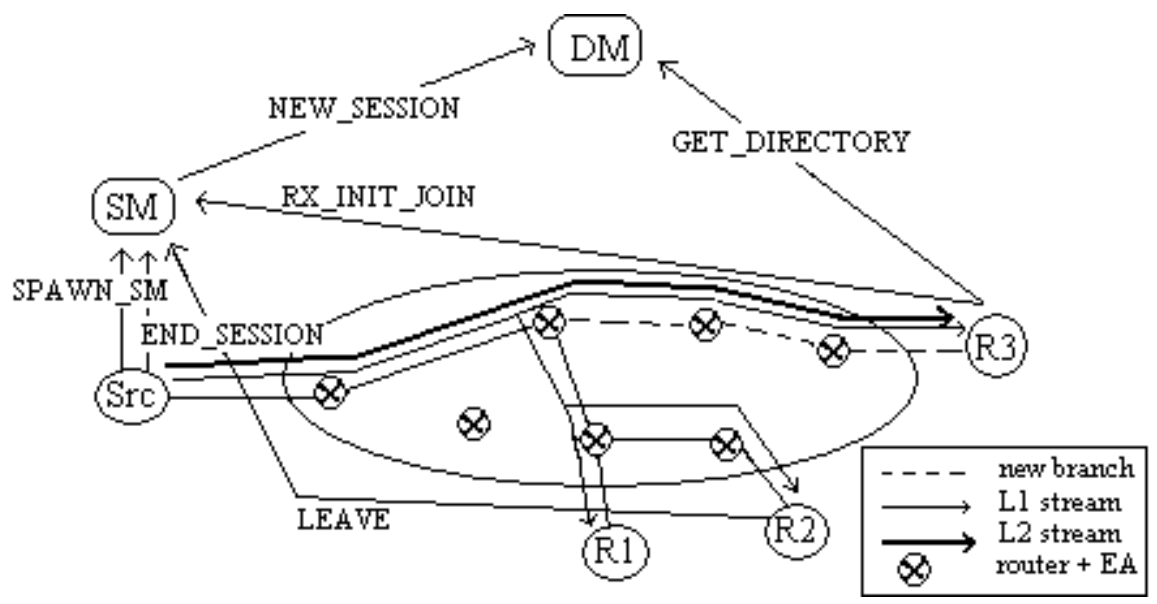

Fig. 4. Multicasting Layered Video over an MRMA-Managed Network

Create Session. Before multicasting can begin Src must spawn a session manager, $\mathrm{SM}$, to initialize and register the session. Src also informs SM of desired sessionspecific QoS parameters such as maximum/minimum source rate (i.e., L1...L4 transmission rates), maximum number of group members, session duration, and etc. SM sends a NEW_SESSION message to DM to negotiate the QoS parameters on behalf of Src. DM makes the session admission decision based on the current network state (i.e., congestion level and available network resource level), and domain policies. If admitted, DM invokes the Address Manager to assign an unique multicast address to the session. DM then registers the new session with DM's Directory Manager so that its presence can be advertised to the entire network. The multicast session is now operational. Next, SM automatically invites any receivers that the source is interested in initiating multicasting with, if any. Otherwise, SM waits for new receivers to join.

Add New Members. We now assume the group already has two members, R1 and R2, and $\mathrm{Src}$ is multicasting layer L1 video to both. To join the group, the new receiver R3 first queries DM's Directory database for various session properties, including the multicast address and SM's IP address. R3 then sends a RX_INIT_JOIN message to SM containing receiver-specified QoS parameters (i.e., R3 wants to receive L1 and L2 layers). 
Adding a new member requires SM to conduct admission-control and connection setup for a new tree branch. A new tree branch joining the new member to the tree would be part of the path, Path(Src, R3). Upon receiving the RX_INIT_JOIN message, SM first verifies if the receiver-specified QoS metrics satisfy session policies (i.e., there may be a policy that restricts non-local-domain receivers from joining multicast sessions that have a local-domain scope).

Next, the SM invokes the Multicast Route Manager to compute a feasible path connecting the existing tree to R3. The computation is based on state information from the Domain Topology and Tree Topology images, and the constraints defined by the receiver-specified QoS metrics. The selected route must have enough free bandwidth for transmitting the L1 and L2 streams. Once a feasible path is identified, $\mathrm{SM}$ attempts to reserve appropriate resources from all on-path nodes from $\mathrm{Path}(\mathrm{Src}$, R3). SM uses source-routing to send a RESERVE mobile agent that traverses the path from the Src to R3. The mobile agent can be a simple control packet or a control script. For each node it traverses, the mobile agent attempts to ensure the required resources are reserved and the multicast routing table is updated to reflect the new receiver. The affected nodes will include existing on-tree nodes and nodes comprising the new branch. If for some reason the state of any of the on-path nodes change during this process and it fails to reserve the required resources at a particular node, the SM executes a 'crank-back' mechanism [5] to release already reserved resources along the path. It can then invoke the Multicast Route Manager to recompute an alternate route based on the new state information. Once path reservation succeeds, the join request is accepted and R3 can begin receiving multicast traffic. However, the join request is rejected if any of the above steps fail. Src now can begin multicasting both L1 and L2 streams, but only R3 will receive both streams.

The Source-initiated Join operation is very similar to the Receiver-initiated Join operation except SM must first invite a receiver to join.

Remove Members. With reference to Fig. 4, R2 wants to leave the group and so it must inform SM with a LEAVE message. Upon receiving the request SM queries its Tree Topology database to identify the tree branch that connects R2. To prune the branch, SM reverses the branch grafting operation. SM uses source-routing to transmit a RECLAIM mobile agent that will traverse along the tree from the source to the departing receiver. Upon reaching $\mathrm{R} 2$, the agent then propagates upstream along the reverse-path back towards SM. Along the way, it will deallocate the appropriate resources and remove the entry for $\mathrm{R} 2$ from the multicast routing table of each node it traverses. By reclaiming resources in a 'backwards' order fashion towards the source, we avoid the danger of pruning a branch too prematurely i.e. removing a branch entry from a upstream node's routing table before having the chance to successfully reclaim resources from all the downstream nodes.

Terminate Session. A multicast session can be terminated either by DM or the source. Upon receiving an END_SESSION message, SM terminates the session by removing all the group members, deallocating from the on-tree nodes all the resources that were reserved for the multicast session, removing the session entry from their multicast routing tables, unregistering the session from the DM's Session Directory, and finally killing the SM process. Much of this procedure is again conducted by multicasting a RECLAIM mobile agent through the tree. 


\section{Conclusions}

In this paper we introduced MRMA, a hierarchical multicast management architecture that is designed to provision multicast-with-QoS service over IP networks. Our architecture integrates all the key multicast functions (route selection, address allocation, session advertisement, congestion and transport control, tree monitoring and tree construction) into a single integrated management framework. It features a centralized monitoring mechanism that allows accurate gathering of domain topology information. It also introduces the concept of the Session Manager which allows multicast sessions to be managed on a per-session basis and distribute the aggregate computational load outside the network and onto the hosts. Together the two mechanisms result in good management accuracy and scalability. Furthermore, the architecture models each of the above multicast components as stand-alone functional blocks that embed into a kernel core. The model allows the flexibility to 'swap-in' and 'swap-out' different implementations for each of the functional blocks, which facilitates leveraging of existing solutions.

For the next phase of our work, we plan to implement a MRMA prototype that can provision layered video multicast applications. In the near future, multimedia applications that require distribution of video over the Internet, such as video conferencing and video-on-demand, are good 'killer application' candidates, and multicasting the video in multiple layered streams is an effective technique for improving the fairness among the receivers. We hope to evaluate MRMA's support mechanisms for constructing multicast trees, for reserving resources and establishing QoS bounds on end-to-end delay, jitter variance, and data loss, and for scaling to accommodate growth. Overall, we hope to evaluate the architecture's overall ability to provision multicast-with-QoS service.

\section{References}

[1] S. Deering and D. Cheriton, "Multicast routing in datagram internetworks and extended LANs," ACM Transactions on Computer Systems, pp.85-111, May 1990.

[2] H. Eriksson, "The Multicast Backbone," Commun. ACM, vol. 8, 1994, pp. 5460.

[3] S. Chen and K. Nahrstedt, "An Overview of Quality of Service Routing for NextGeneration High-Speed Networks: Problems and Solutions," IEEE Network, Nov/Dec. 1998.

[4] H. Holbrook and D. Cheriton, "IP Multicast Channels: EXPRESS Support for Large-Scale Single-Source Applications,: ACM SIGCOMM, Cambridge, MA, Aug. 1999.

[5] Ryan Wu, Irene Katzela, "Multicast Resource Management Architecture", University of Toronto, 2000.

[6] A. Banerjea, R. Pankaj, M. Foloutsos, "QoSMIC: Quality of Service sensitive Multicast Internet protoCol”, SIGCOMM'98, 1998. 